\title{
Factores que influyen en el uso de praderas cultivadas para producción de leche en pequeña escala en el altiplano central mexicano
}

\section{Factors influencing the use of cultivated grassland for small- scale dairy production in the Central Highlands of Mexico}

\author{
Marilyn Juárez-Moralesa, Carlos Manuel Arriaga-Jordánª, Ernesto Sánchez-Veraa, Juan de Dios García- \\ Villegas ${ }^{a}$, Adolfo Armando Rayas-Amorb, Tahir Rehmanc, Peter Dorwardc, Carlos Galdino Martínez-García ${ }^{\text {a* }}$
}

\section{RESUMEN}

El objetivo fue identificar variables que describen al productor y a la unidad de producción, así como factores cognoscitivos y sociales que influyen en el uso de praderas cultivadas por productores de leche en pequeña escala. La muestra $(n=106)$ se dividió en productores que hacen uso de la innovación (Grupo $1, n=55)$ y en productores que no la usan (Grupo 2, $n=51$ ). Los datos se analizaron con la prueba de Mann Withney y la Teoría del Comportamiento Planeado. El uso de praderas cultivadas se vio influenciado por las variables que describen al productor $\mathrm{y}$ a la unidad de producción, como se observó en el Grupo 1. Este grupo presentó una mayor proporción de productores (60 \%) que indicaron una intención positiva para usar praderas cultivadas, comparado con el $36 \%$ de los productores del Grupo 2. Los productores de ambos grupos percibieron de forma similar la utilidad e importancia del uso de praderas cultivadas; sin embargo, la intención de los productores del Grupo 1 presentó una correlación significativa $(P<0.05)$ con ambas. Otros factores que influyeron en el uso de praderas cultivadas fueron las creencias positivas, referentes sociales y las dificultades percibidas de la innovación. Se concluye que el uso de praderas cultivadas estuvo influenciado por diferentes factores para cada grupo de productores.

PALABRAS CLAVE: Teoría del comportamiento planeado (TPB), Praderas cultivadas, Productores de leche.

\section{ABSTRACT}

This paper identifies the socio-economic and farm variables and cognitive and social factors influencing the use of cultivated pastures by small-scale dairy farmers. A field survey was conducted. The sample was divided in two groups defined as: users (Group 1, $n=55$ ) and non-users of the innovation (Group 2, $n=51$ ). Data were analysed through the Mann Whitney test and the Theory of Planned Behaviour (TPB). Socioeconomic and farm variables influenced the use of cultivated grassland, as observed in Group 1. This group showed a bigger proportion of farmers $(60 \%)$ who indicated a positive intention to use cultivated grasslands, in comparison with $36 \%$ of farmers from Group 2. Farmers from both groups perceived similarly the utility and importance of the use of cultivated grassland; however, farmers' intention of Group 1 showed a significant correlation $(P<0.05)$ with utility and importance. Other factors influencing the use of cultivated grasslands were the positive beliefs, social referents and difficulties perceived of the innovation. It concludes that different factors influenced the use of cultivated grassland in each group.

KEY WORDS: Theory of planned behaviour (TPB), Cultivated pastures, Dairy producers.

Recibido el 15 de marzo de 2016. Aceptado el 28 de abril de 2016.

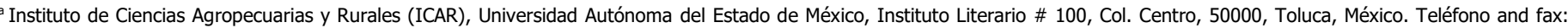
00527222965552.

${ }^{b}$ Departamento de Ciencias de la Alimentación, División de Ciencias Biológicas y de la Salud, Universidad Autónoma Metropolitana. Estado de México, México.

'School of Agriculture, Policy and Development, University of Reading. Reading, UK.

*Autor de correspondencia: cgmartinezg@uaemex.mx. 
En México, los sistemas de producción de leche en pequeña escala (SPLPE) generan empleos e ingresos diarios y son considerados como una alternativa de desarrollo rural ${ }^{(1)}$. La problemática que presentan estos sistemas son los altos costos de producción ${ }^{(2)}$ y la baja adopción de innovaciones que brindan mayor sustentabilidad económica, como son las praderas cultivadas ${ }^{(3)}$. Existen estudios indican que las variables que describen al productor y a la unidad de producción influyen en la adopción de innovaciones ${ }^{(4,5)}$. Sin embargo, estos estudios no han considerado factores cognoscitivos (creencias) y socio-psicológicos (referentes sociales), que influyen en la toma de decisiones del productor.

En el contexto internacional, se han identificado creencias y referentes sociales a partir de la aplicación de la Teoría de la acción razonada (TRA, por sus siglas en inglés) y la Teoría del comportamiento planeado (TPB, por sus siglas en inglés), los cuales han ayudado a entender la toma de decisiones de los productores en la adopción o rechazo de innovaciones ${ }^{(6,7)}$. En el contexto mexicano, dichos modelos son herramientas de investigación innovadoras, que recientemente han sido utilizadas para estudiar factores que influyen en la adopción de innovaciones en los SPLPE ${ }^{(8)}$. Por lo tanto, el objetivo del estudio fue identificar variables que describen al productor y a la unidad de producción, así como factores cognoscitivos y sociales que influyen en el uso de praderas cultivadas por productores de leche en pequeña escala del altiplano central mexicano.

Para el desarrollo de este estudio se utilizó el modelo socio-psicológico de la TPB ${ }^{(9)}$. Este modelo explica que la toma de decisiones está determinada por la intención del individuo, la cual es un indicador de la disposición del individuo para llevar a cabo un determinado comportamiento, y se considera que es el antecedente inmediato de la conducta ${ }^{(9)}$. La intención es considerada como una variable dependiente, la cual está influenciada por los tres componentes de la TPB: actitud (creencias conductuales), norma subjetiva (referentes sociales) y control conductual percibido (dificultades percibidas) $)^{(8)}$.

Los datos para el modelo se colectaron en dos fases. En la primera se realizaron 40 entrevistas semiestructuradas, 20 con productores que usan praderas cultivadas y 20 que no usan la innovación. Las entrevistas permitieron obtener 10 creencias, 7 referentes sociales y 4 dificultades. En la segunda fase, se elaboró un cuestionario estructurado que incluyó las creencias, los referentes sociales y las dificultades identificadas en la primera fase. El cuestionario también colectó información sobre las características del productor y de la unidad de producción.

El cuestionario se aplicó a 106 productores de leche en pequeña escala del municipio de Aculco, Estado de México, los cuales representan el $10 \%$ de la población total del área de estudio. Los datos se colectaron durante los meses de febrero a junio de 2013. La identificación de los productores se realizó a través de un muestreo no probabilístico conocido como "bola de nieve"(10).

La muestra total $(n=106)$ se dividió en productores que hacen uso de praderas cultivadas (Grupo $1, \mathrm{n}=55$ ) y en productores que no hace uso de la innovación (Grupo 2, n= 51), con el propósito de identificar diferencias entre grupos, con respecto a las variables que describen a las características del productor (edad, educación y experiencia) y características de la unidad de producción (miembros de la familia, mano de obra familiar, tamaño de hato, vacas en producción, producción de leche por vaca por día, leche vendida por día, precio de la leche, número total de hectáreas y área utilizada para pradera).

Las 12 variables analizadas no presentaron distribución normal con la prueba de KolmogorovSmirnov ${ }^{(11)}$; por lo cual, para identificar diferencias entre grupos, se utilizó la prueba no paramétrica de Mann-Withney ${ }^{(11)}$, considerando diferencias a $P<0.05$. La mediana y el rango intercuartil se utilizaron como medida de tendencia central y dispersión ${ }^{(11)}$. Se realizó un análisis de correlaciones no paramétricas de Spearman $(P<0.05)$ para identificar variables que tuvieran correlación con la intención de los productores para el uso de praderas cultivadas.

Los tres componentes del modelo TPB $\left(A=\Sigma b_{i}\right.$ $\left.e_{i} ; S N=\Sigma s b_{j} m_{j} ; P B C=\Sigma c_{k} p_{k}\right)$ se midieron a través de una escala unipolar de cinco puntos tipo Likert ${ }^{(12)}$. 
Así mismo, el análisis de la información se realizó a través de correlaciones de Spearman ${ }^{(11)}$. Para identificar diferencias entre grupos con respecto a los tres componentes del modelo se utilizó la prueba de Mann Whitney ${ }^{(11)}$. Se utilizó la mediana y el rango intercuartil (IQR) como medidas de tendencia central y de dispersión. Para medir la confiabilidad de la escala utilizada, se utilizó el coeficiente a de Cronbach. Los datos se analizaron con el programa estadístico SPSS versión 22.

Los resultados muestran que ambos grupos presentaron diferencias $(P<0.05)$ en dos de las tres variables que describen a las características del productor, y en seis de nueve variables que describen a las características de la unidad de producción (Cuadro 1). La intención de los productores para utilizar praderas cultivadas en los próximos 12 meses no estuvo correlacionada con las variables que describen al productor y la unidad de producción en ambos grupos; sin embargo, la intención de los productores del Grupo 2, solo presentó una correlación positiva $(P<0.05)$ con la variable precio de la leche.

La prueba de Mann Whitney $U$ mostró que la intención de usar praderas cultivadas en los siguientes 12 meses por los productores del Grupo 1 no presentó diferencias $U=1165$ ( $P>0.05)$ con respecto a la intención de los productores del Grupo 2. La percepción de la utilidad de la innovación, no mostró diferencias ( $P>0.05$ ) entre ambos grupos. Sin embargo, la utilidad que perciben los productores del Grupo 1, presentó una mediana correlación $(r=0.699, P<0.001)$ con su intención.

La actitud directa presentó una mediana correlación significativa $(r=0.667, P<0.001)$ con la intención de los productores del Grupo 1. Con respecto a la medición de la actitud indirecta (Cuadro 2) podemos observar que en el resultado de la evaluación $\left(\mathrm{e}_{\mathrm{i}}\right)$, la creencia: "el uso de praderas cultivadas requiere poco trabajo y una mínima inversión para su mantenimiento", presentó diferencias $(P<0.05)$, siendo mejor evaluada por los productores del Grupo 2. Así mismo la suma de los productos que indican la actitud general mostró diferencias $(P<0.05)$ entre ambos grupos; siendo mejor evaluada por los productores del Grupo 1.

Se identificaron cinco creencias que actúan como conductores en ambos grupos, las cuales fueron: a) el uso de praderas cultivadas permite tener forraje de buena calidad y de bajo costo, b) aumenta la producción de leche, $\mathrm{c}$ ) reduce los gastos de alimentación en la época de lluvia, d) requieren

Cuadro 1. Características generales y diferencia entre grupos de productores

\begin{tabular}{lccccc}
\hline Variables & \multicolumn{2}{c}{$\begin{array}{c}\text { Usan praderas } \\
(\mathrm{n}=55)\end{array}$} & \multicolumn{2}{c}{$\begin{array}{c}\text { No usan praderas } \\
(\mathrm{n}=51)\end{array}$} & $P$ \\
\hline Características del productor: & Mediana & RIC & Mediana & RIC & \\
Edad, años & 49 & 20 & 42 & 21 & $<0.029$ \\
Educación, años & 6 & 3 & 6 & 4 & 0.879 \\
Experiencia, años & 26 & 20 & 20 & 20 & $<0.006$ \\
Características de la unidad de producción: & & & & & \\
Miembros de la familia, personas & 5 & 2 & 4 & 2 & 0.149 \\
Mano de obra familiar, personas & 2 & 2 & 2 & 3 & 0.070 \\
Tamaño de hato, animales & 15 & 10 & 6 & 7 & $<0.000$ \\
Vacas en producción, animales & 8 & 7 & 3 & 4 & $<0.000$ \\
Producción leche/vaca/día, L & 15 & 8 & 12 & 7 & $<0.002$ \\
Leche vendida por día, L & 120 & 128 & 36 & 58 & $<0.000$ \\
Precio leche, pesos mexicanos & 5.2 & 0.4 & 5.0 & 0.2 & $<0.039$ \\
Número total de hectáreas & 5.0 & 6.0 & 1.5 & 2.8 & $<0.000$ \\
Área utilizada para pradera, ha & 1.0 & 1.0 & 0.0 & 0.0 & -- \\
\hline
\end{tabular}

$\mathrm{RIC}=$ rango intercuartil; $P=$ valor de la prueba de Mann Whithney $U(P<0.05)$. 
Cuadro 2. Medianas y rango intercuartil () de la comparación del resultado de las creencias $\left(b_{i}\right)$, resultado de la evaluación $\left(e_{i}\right)$ y actitud $\left(b_{i}^{*} e_{i}\right)$

\begin{tabular}{|c|c|c|c|c|c|c|c|c|c|}
\hline \multirow{2}{*}{$\begin{array}{l}\text { Creencias } \\
\text { El uso de praderas cultivadas } \\
\text { en la unidad de producción }\end{array}$} & \multicolumn{3}{|c|}{$\begin{array}{l}\text { Resultado de la creencia } \\
\left(\mathrm{b}_{\mathrm{i}}\right)\end{array}$} & \multicolumn{3}{|c|}{$\begin{array}{l}\text { Resultado de la valuación } \\
\left(\mathrm{e}_{\mathrm{i}}\right)\end{array}$} & \multirow{2}{*}{$\begin{array}{l}\text { Actitud } \\
\left(b_{i}^{*} e_{i}\right) \\
\text { Usan } \\
\text { praderas }\end{array}$} & \multirow[b]{2}{*}{$\begin{array}{l}\text { No usan } \\
\text { praderas }\end{array}$} & \multirow[b]{2}{*}{$P$} \\
\hline & $\begin{array}{l}\text { Usan } \\
\text { praderas }\end{array}$ & $\begin{array}{l}\text { No usan } \\
\text { praderas }\end{array}$ & $P$ & $\begin{array}{l}\text { Usan } \\
\text { praderas }\end{array}$ & $\begin{array}{l}\text { No usan } \\
\text { praderas }\end{array}$ & $P$ & & & \\
\hline \multicolumn{10}{|l|}{ VENTAJAS } \\
\hline Tener forraje de buena calidad & $4.5(1.0)$ & $5.0(1.0)$ & 0.645 & $4.0(1.0)$ & $4.0(1.0)$ & 0.883 & $20.0(9.0)$ & $20.0(9.0)$ & 0.512 \\
\hline $\begin{array}{l}\text { Permite tener forraje a bajo } \\
\text { costo }\end{array}$ & $5.0(1.0)$ & $5.0(1.0)$ & 0.727 & $5.0(1.0)$ & $5.0(1.0)$ & 0.511 & $20.0(9.0)$ & $20.0(9.0)$ & 0.852 \\
\hline $\begin{array}{l}\text { Aumento de la producción de } \\
\text { leche }\end{array}$ & $5.0(1.0)$ & $5.0(1.0)$ & 0.944 & $5.0(1.0)$ & $5.0(1.0)$ & 0.543 & $20.0(10.0)$ & $20.0(10.0)$ & 0.961 \\
\hline $\begin{array}{l}\text { Reduce gastos de } \\
\text { alimentación en época de } \\
\text { lluvias }\end{array}$ & $4.0(2.0)$ & $4.0(1.0)$ & 0.387 & $4.0(2.0)$ & $5.0(1.0)$ & 0.349 & $16.0(13.0)$ & $20.0(10.0)$ & 0.281 \\
\hline $\begin{array}{l}\text { Requiere poco trabajo y } \\
\text { mínima inversión para su } \\
\text { mantenimiento }\end{array}$ & $5.0(2.0)$ & $5.0(1.0)$ & 0.462 & $4.0(2.0)$ & $5.0(1.0)$ & $<0.035$ & $16.0(13.0)$ & $20.0(10.0)$ & 0.119 \\
\hline $\begin{array}{l}\text { Permite uso de estiércol } \\
\text { producido en la granja }\end{array}$ & $5.0(1.0)$ & $5.0(1.0)$ & 0.970 & $4.0(1.0)$ & $5.0(1.0)$ & 0.190 & $20.0(9.0)$ & $20.0(9.0)$ & 0.448 \\
\hline $\begin{array}{l}\text { No requiere de tiempo y mano } \\
\text { de obra cuando se utiliza para } \\
\text { pastoreo }\end{array}$ & $4.0(2.0)$ & $5.0(2.0)$ & 0.562 & $4.0(1.0)$ & $5.0(1.0)$ & 0.564 & $20.0(13.0)$ & $20.0(13.0)$ & 0.445 \\
\hline \multicolumn{10}{|l|}{ DESVENTAJAS } \\
\hline $\begin{array}{l}\text { Requiere de varios riegos } \\
\text { durante el invierno y primavera }\end{array}$ & $5.0(1.0)$ & $5.0(1.0)$ & 0.845 & $5.0(1.0)$ & $4.0(1.0)$ & 0.699 & $20.0(9.0)$ & $20.0(9.0)$ & 0.809 \\
\hline $\begin{array}{l}\text { Ofrece poca disponibilidad de } \\
\text { forraje durante el invierno }\end{array}$ & $5.0(1.0)$ & $5.0(1.0)$ & 0.771 & $4.0(1.0)$ & $5.0(1.0)$ & 0.455 & $20.0(9.0)$ & $25.0(9.0)$ & 0.702 \\
\hline $\begin{array}{l}\text { Requieren de mayor superficie } \\
\text { de tierra para alimentar a todo } \\
\text { el hato }\end{array}$ & $5.0(1.0)$ & $5.0(1.0)$ & 0.406 & $5.0(1.0)$ & $5.0(1.0)$ & 0.419 & $25.0(9.0)$ & $25.0(9.0)$ & 0.392 \\
\hline$\sum \mathrm{b}_{\mathrm{i}}$ & $45.0(7.0)$ & $45.0(7.0)$ & 0.903 & & & & & & \\
\hline$\sum \mathrm{e}_{\mathrm{i}}$ & & & & $43.0(10.0)$ & $46.0(10.0)$ & 0.493 & & & \\
\hline$\sum b_{i}^{*} e_{i}$ & & & & & & & $194.0(72.0)$ & $187.0(63.0)$ & $<0.030$ \\
\hline Coeficiente $\alpha$ de Cronbrach & & & & & & & & & 0.747 \\
\hline
\end{tabular}

de poco trabajo y una mínima inversión para su mantenimiento. Por otro lado, se encontraron dos creencias que actúan como barrera, las cuales fueron: a) el uso de praderas cultivadas requiere de mayor superficie de tierra para alimentar a todo el hato y b) requiere de varios riegos durante el invierno y primavera.

La norma subjetiva directa presentó una correlación baja ( $r=0.457, P<0.001 ;(r=0.326$, $P<0.001)$ con la intención de los productores del Grupo 1 y Grupo 2, respectivamente. La medición de la norma subjetiva indirecta (Cuadro 3) indica que de los siete referentes sociales identificados, únicamente el vendedor de forrajes presentó el menor puntaje por ambos grupos; es decir, es el referente social con el cual los productores se sentirían no muy animados y no del todo motivados para usar praderas cultivadas en los siguientes 12 meses.

El control conductual percibido directo presentó una correlación baja $(r=0.551, P<0.001 ;(r=0.318$, $P<0.05)$ con la intención de los productores del Grupo 1 y Grupo 2, respectivamente. El control conductual percibido indirecto identificó cuatro dificultades asociadas con el uso de praderas cultivadas (Cuadro 4). En las puntuaciones del control de las creencias $\left(\mathrm{cc}_{\mathrm{k}}\right)$, se puede observar que 
los productores de ambos grupos están de acuerdo en que la compra de semilla, pagar el servicio de riego, y la inversión inicial no se recuperan con la venta de la leche, y que la falta de asesoría y conocimiento para su uso, son factores que dificultan el uso de praderas cultivadas en los siguientes 12 meses; sin embargo, los productores manifestaron facilidad para poder controlar las dificultades percibidas, como se observa en los puntajes del poder del control de las creencias $\left(p_{k}\right)$.

Cuadro 3. Medianas y rango intercuartil () de la comparación de la creencia subjetiva $\left(s b_{j}\right)$, motivación para cumplir $\left(\mathrm{m}_{\mathrm{j}}\right)$ y la norma subjetiva $\left(m_{j}^{*} s b_{j}\right)$

\begin{tabular}{|c|c|c|c|c|c|c|c|c|c|}
\hline \multirow[b]{2}{*}{ Referentes sociales } & \multicolumn{2}{|c|}{$\begin{array}{c}\text { Creencia subjetiva } \\
\left(s b_{j}\right)\end{array}$} & \multirow[b]{2}{*}{$P$} & \multicolumn{2}{|c|}{$\begin{array}{c}\text { Motivación para cumplir } \\
\left(\mathrm{m}_{\mathrm{j}}\right)\end{array}$} & \multicolumn{4}{|c|}{$\begin{array}{c}\text { Norma subjetiva } \\
\left(m_{j}^{*}{ }^{*} b_{j}\right)\end{array}$} \\
\hline & $\begin{array}{l}\text { Usan } \\
\text { pradera }\end{array}$ & $\begin{array}{l}\text { No usan } \\
\text { pradera }\end{array}$ & & $\begin{array}{l}\text { Usan } \\
\text { pradera }\end{array}$ & $\begin{array}{l}\text { No usan } \\
\text { pradera }\end{array}$ & $P$ & $\begin{array}{l}\text { Usan } \\
\text { pradera }\end{array}$ & $\begin{array}{l}\text { No usan } \\
\text { pradera }\end{array}$ & $P$ \\
\hline Otros productores & $4.0(1)$ & $4.0(1)$ & 0.980 & $4.0(1)$ & $4.0(1)$ & 0.980 & $16.0(11)$ & $12.0(10)$ & 0.520 \\
\hline $\begin{array}{l}\text { Prestadores de } \\
\text { profesionales }\end{array}$ & $4.0(1)$ & $4.0(2)$ & 0.526 & $4.0(1)$ & $4.0(2)$ & 0.526 & $12.0(7)$ & $12.0(11)$ & 0.879 \\
\hline Vendedor de forrajes & $2.0(2)$ & $2.0(3)$ & 0.150 & $2.0(2)$ & $2.0(1)$ & 0.150 & $4.0(8)$ & $5.0(11)$ & 0.093 \\
\hline Universidad & $4.0(2)$ & $4.0(1)$ & 0.685 & $4.0(1)$ & $4.0(1)$ & 0.685 & $16.0(13)$ & $12.0(8)$ & 0.873 \\
\hline Padre & $4.0(1)$ & $4.0(2)$ & 0.537 & $4.0(2)$ & $4.0(2)$ & 0.537 & $16.0(11)$ & $10.5(12)$ & 0.345 \\
\hline Hermano & $4.0(1)$ & $4.0(2)$ & 0.309 & $4.0(2)$ & $4.0(2)$ & 0.309 & $16.0(10)$ & $12.0(13)$ & 0.213 \\
\hline Tío & $4.0(1)$ & $4.0(2)$ & 0.474 & $4.0(1)$ & $4.0(2)$ & 0.474 & $16.0(10)$ & $12.0(12)$ & 0.311 \\
\hline$\sum s b_{j}$ & $19.0(6)$ & $21.0(5)$ & 0.532 & & & & & & \\
\hline$\sum m_{j}$ & & & & $24.0(8)$ & $23.0(8)$ & .612 & & & \\
\hline$\sum \mathrm{sbj}_{j}^{*} \mathrm{~m}_{\mathrm{j}}$ & & & & & & & $76.0(42)$ & $78.0(55)$ & 0.374 \\
\hline Coeficiente $\alpha$ de Cronbrach & & & & & & & & & 0.758 \\
\hline
\end{tabular}

subjetiva $\left(m_{j}^{*} s b_{j}\right)$

$P=$ valor de la prueba de $U$ de Mann Whitney $(P<0.05)$.

Cuadro 4. Mediana y rango intercuartil () de la comparación del control de la creencia ( $\left.c c_{k}\right)$, poder de control de la creencia $\left(p_{k}\right)$ y el control conductual percibido $\left(\mathrm{cc}_{\mathrm{k}}{ }^{*} \mathrm{p}_{\mathrm{k}}\right)$

\begin{tabular}{|c|c|c|c|c|c|c|c|c|c|}
\hline \multirow[b]{2}{*}{$\begin{array}{l}\text { Dificultades percibidas } \\
\text { para el uso de praderas }\end{array}$} & \multicolumn{2}{|c|}{$\begin{array}{c}\text { Control de la creencia } \\
\left.\text { ( } \mathrm{cc}_{\mathrm{k}}\right)\end{array}$} & \multirow[b]{2}{*}{$P$} & \multicolumn{2}{|c|}{$\begin{array}{l}\text { Poder de control de la } \\
\text { creencia }\left(p_{k}\right)\end{array}$} & \multirow[b]{2}{*}{$P$} & \multicolumn{2}{|c|}{$\begin{array}{l}\text { Control conductual } \\
\text { percibido }\left(\mathrm{cc}_{k}^{*} \mathrm{p}_{k}\right)\end{array}$} & \multirow[b]{2}{*}{$P$} \\
\hline & $\begin{array}{l}\text { Usan } \\
\text { praderas }\end{array}$ & $\begin{array}{l}\text { No usan } \\
\text { praderas }\end{array}$ & & $\begin{array}{l}\text { Usan } \\
\text { praderas }\end{array}$ & $\begin{array}{l}\text { No usan } \\
\text { praderas }\end{array}$ & & $\begin{array}{l}\text { Usan } \\
\text { praderas }\end{array}$ & $\begin{array}{l}\text { No usan } \\
\text { praderas }\end{array}$ & \\
\hline Compra de semilla & $4.0(2)$ & $4.0(2)$ & 0.549 & $4.0(1)$ & $4.0(2)$ & 0.549 & $15.0(7)$ & $12.0(8)$ & 0.269 \\
\hline Pago servicio de riego & $4.0(2)$ & $4.0(1)$ & 0.874 & $4.0(1)$ & $4.0(2)$ & 0.435 & $15.0(12)$ & $16.0(16)$ & 0.851 \\
\hline $\begin{array}{l}\text { Inversión irrecuperable } \\
\text { con venta de leche }\end{array}$ & $5.0(1)$ & $4.0(2)$ & 0.486 & $4.0(1)$ & $4.0(2)$ & 0.802 & $15.0(8)$ & $15.0(8)$ & 0.987 \\
\hline $\begin{array}{l}\text { Falta de asesoría para } \\
\text { su uso }\end{array}$ & $4.0(2)$ & $4.0(2)$ & 0.787 & $4.0(2)$ & $4.0(2)$ & 0.299 & $15.0(8)$ & $15.0(12)$ & 0.766 \\
\hline$\sum \mathrm{CC}_{k}$ & $16.0(6)$ & $16.0(5)$ & 0.439 & & & & & & \\
\hline$\sum p_{k}$ & & & & $14.0(6)$ & $15.0(6)$ & 0.164 & & & \\
\hline$\sum c c_{k} \times p_{k}$ & & & & & & & $60.0(37)$ & $62.0(37)$ & 0.492 \\
\hline $\begin{array}{l}\text { Coeficiente } \alpha \text { de } \\
\text { Cronbrach }\end{array}$ & & & & & & & & & 0.784 \\
\hline
\end{tabular}

$P=$ valor de la prueba de $U$ de Mann Whitney $(P<0.05)$. 
Los resultados indican que las variables que describen al productor (edad y experiencia) y a la unidad de producción (tamaño de hato, vacas en producción, producción de leche por vaca por día, cantidad de leche vendida por día, precio por litro de leche y extensión de tierra); juegan un papel importante en el uso de praderas cultivadas ${ }^{(10)}$ como se observó en el Grupo 1. Se ha observado que la disponibilidad de tierra es una variable fundamental en el uso de praderas cultivadas ${ }^{(13)}$.

La correlación positiva $(P<0.05)$ entre la intención de los productores del Grupo 2 y el precio de la leche, indica que los productores que reciben un mejor pago por litro de leche, tienen una mayor intención para hacer uso de praderas cultivadas. La intención de los productores del Grupo 1 para usar praderas cultivadas fue mayor a la del Grupo 2. Esto puede atribuirse a la experiencia y conocimiento que tienen los productores del Grupo 1, con respecto al uso de la innovación. Así mismo, la correlación positiva $(P<0.05)$ de la intención de los productores del Grupo 1, con la utilidad e importancia del uso de praderas cultivadas, sugiere que entre mayor sea la utilidad e importancia percibida, más fuerte será la intención de los productores para usar la innovación en los próximos 12 meses en la unidad de producción.

La actitud indirecta, indicó que las creencias se evaluaron de forma semejante por los productores de ambos grupos; sin embargo, las diferencias $(P<0.05)$ observadas con respecto a la creencia: las praderas cultivadas requieren poco trabajo y mínima inversión para su mantenimiento, sugiere que los productores del Grupo 2 podrían considerar a la innovación como una alternativa dentro de su unidad de producción, ya que los productores están más dispuestos a adoptar innovaciones que son fáciles de implementar y que tienen beneficios inmediatos $^{(14)}$.

Las diferencias observadas $(P<0.05)$ entre grupos con respecto a la actitud general, muestra que el uso de praderas cultivadas está influenciada en mayor medida en el Grupo 1, por las creencias positivas y negativas de la innovación, que pueden ser consideradas como conductores y barreras, las cuales son creencias positivas y negativas que favorecen o limitan el uso o adopción de la innovación ${ }^{(8)}$. Las cinco creencias identificadas como conductores en ambos grupos, podrían ser utilizadas por servicios de extensión para la promoción y comunicación de la innovación ${ }^{(8,15)}$.

Las dos mediciones de la norma subjetiva indican que la intención y la toma de decisiones de los productores de ambos grupos, pueden estar influenciadas por sus referentes sociales tales como: otro productor, prestador de servicios profesionales, universidad, padre, hermano y tío. Se ha identificado que el padre y abuelo juegan un papel importante en la comunicación para el uso de praderas cultivadas ${ }^{(8)}$; así mismo los referentes sociales más cercanos como familiares, amigos y productores vecinos fueron los referentes más importantes $^{(15)}$. Se indica que las redes sociales de familiares, amigos y grupo de productores son avenidas importantes para la diseminación de nuevas innovaciones ${ }^{(14,16)}$. Por lo tanto, los referentes sociales identificados en este estudio, deberían ser considerados como la principal fuente de comunicación y difusión de la innovación hacia los productores ${ }^{(7,8,15)}$. Por otro lado, el vendedor de forrajes fue identificado como un referente social irrelevante; sin embargo, este referente social jugó un papel importante en la comunicación y difusión de praderas cultivadas ${ }^{(15)}$.

La medición del control conductual percibido directo, muestra que los productores de ambos grupos indicaron dificultad para usar praderas cultivadas en los siguientes 12 meses. La dificultad percibida por los productores del Grupo 1 podría estar atribuida a la disminución de forraje durante la época de invierno y a la falta de agua para los riegos durante la época de secas $^{(8)}$; mientras que para los productores del Grupo 2 pudiera estar asociada a la falta de experiencia y a la poca disponibilidad de tierra(8).

La medición del control conductual percibido general no presentó diferencias ( $P>0.05)$, lo que indica que ambos grupos consideran que la compra de semilla, realizar el pago del riego, la inversión inicial no se recupera con la venta de la leche, la falta de asesoría y falta de conocimiento para su uso, son creencias que dificultan y limitan el uso de praderas cultivadas. Las tres primeras creencias están enfocadas al aspecto económico, lo que puede decir que la falta de recursos económicos puede ser 
la mayor limitante para el uso de praderas cultivadas. Así, los productores de leche en pequeña escala con restricción de recursos financieros se ven limitados para implementar nuevas innovaciones ${ }^{(17)}$. Por otro lado, se indica que para incrementar la intención de los productores para usar praderas cultivadas, las organizaciones gubernamentales deberían proporcionar asistencia técnica calificada, lo que permitiría ganar seguridad y conocimiento para el uso de la innovación ${ }^{(15)}$.

Se concluye que los factores que influyen en el uso de praderas cultivadas en los productores del Grupo 1, están relacionados principalmente con la edad y experiencia del productor; así como con el tamaño de hato, vacas en producción, producción de leche por vaca por día, cantidad de leche vendida por día, precio por litro de leche y extensión de tierra (características de la unidad de producción). La intención de los productores para usar praderas cultivadas estuvo influenciada por la utilidad e importancia que los productores perciben de la innovación, como fue el caso del Grupo 1; así mismo, un mejor pago por litro de leche influyó en la intención de los productores del Grupo 2. La intención de los productores de ambos grupos se vio influenciada por las creencias positivas y la presión de los referentes sociales; sin embargo, la intención de los productores del Grupo 2 se vio limitada por las dificultades percibidas de la innovación. Las creencias positivas y referentes sociales identificados en el estudio deberían ser considerados como fuentes de conocimiento y canales de comunicación para brindar servicios de extensión y poder promover el uso de praderas cultivadas.

\section{AGRADECIMIENTOS}

El primer autor agradece al Consejo Nacional de Ciencia y Tecnología (CONACYT) por la beca de maestría y a la Universidad Autónoma del Estado de México por el apoyo brindado para la realización de la investigación.

\section{LITERATURA CITADA}

1. Arriaga-Jordán CM, Albarrán-Portillo B, Espinoza-Ortega A, GarcíaMartínez A, Castelán-Ortega OA. On-farm comparison feeding strategies based on forages for small-scale dairy production systems in the highlands of central Mexico. Expl Agric 2002;38:375388.

2. Espinoza-Ortega A, Álvarez-Macías A, Del Valle MC, Chauvete M. La economía de los sistemas campesinos de producción de leche en el Estado de México. Téc Pecu Méx 2005,43:39-56.

3. Fadul-Pacheco L, Wattiaux MA, Espinoza-Ortega A, SánchezVera $E$, Arriaga-Jordán CM. Evaluation of sustainability of smallholder dairy production systems in the highlands of Mexico during the rainy season. Agroecol Sustainable Food Syst 2013; 37:882-901.

4. Bernués $A$, Herrero $M$. Farm intensification and drivers of technology adoption in mixed dairy-crop systems in Santa Cruz, Bolivia. Span J Agric Res 2008;6:279-293.

5. Martínez-García CG, Dorward $P$, Rehman $T$. Farm and socioeconomic characteristics of small-holder milk producers and their influence on the technology adoption in Central Mexico. Trop Anim Health Prod 2012;44:1199-1211.

6. Rehman T, McKemey K, Yates CM, Cooke RJ, Garforth CJ, Tranter $\mathrm{RB}$, et al. Identifying and understanding factors influencing the uptake of new technologies on dairy farms in SW England using the theory of reasoned action. Agr Syst 2007;94:281-293.

7. Sambodo LAAT, Nuthall PL. A behavioural approach to understanding semi-subsistence farmers' technology adoption decisions: The case of improved paddy-prawn system in Indonesia. J Agric Edu Ext 2010;16:111-129.

8. Martínez-García CG, Dorward P, Rehman T. Factors influencing adoption of improved grassland management by small-scale dairy farmers in Central Mexico and the implications for future research on smallholder adoption in developing countries. Livest Sci 2013;152:228-238.

9. Ajzen I. Attitudes, personality and behaviour. 2nd ed. Milton Keynes, England: Open University Press; 2005.

10. Vogt WP, Burke JR. Dictionary of statistics and methodology: A non-technical guide for the social sciences. 4th ed. USA: Sage Publications; 2011.

11. Field A. Discovering statistics using IBM SPSS Statistics. 4th ed. Great Britain: SAGE Publications; 2013.

12. Bryman A, Cramer D. Quantitative data analysis with IBM SPSS 17, 18 and 19, A Guide for social scientists. London and New York: Routledge, Taylor and Francis Group; 2011.

13. Martínez-García CG, Dorward $P$, Rehman T. Factors influencing adoption of crop and forage related and animal husbandry technologies by small-scale dairy farmers in Central Mexico. Expl Agric 2015;52:87-109.

14. Kiptot E, Franzel S, Hebinck P, Richards P. Sharing seeds and knowledge: farmer to farmer dissemination of agroforestry technologies in western Kenya. Agroforest Syst 2006;68:167-179.

15. Rossi-Borges JA, Oude Lansink AGJM, Marques RC, Lutke V. Understanding farmers' intention to adopt improved natural grassland using the theory of planned behaviour. Livest Sci 2014;169:163-174.

16. Adegbola $P$, Gardebroek $C$. The effect of information sources on technology adoption and modification decision. Agr Econ 2007;37:55-65. 
Marilyn Juárez-Morales, et al. / Rev Mex Cienc Pecu 2017;8(3):317-324

17. Espinoza-Ortega A, Espinosa-Ayala E, Bastida-López J, CastañedaMartínez T, Arriaga-Jordán CM. Small-scale dairy farming in the highlands of central Mexico: Technical, economic and social aspects and their impact on poverty. Expl Agric 2007;43:241-256. 